\title{
Scattering by a Two-dimensional Highly-Conducting Rough Surface
}

\author{
Hongxuan Xu, Yufeng Shao, Jiancun Zuo, Yu Feng, Hong Dai, Liandong Wang, Hua Zhang \\ School of electrical \& electric engineering \\ Shanghai Second Polytechnic University, Shanghai, China, 201209
}

\begin{abstract}
In this paper, we consider an electromagnetic wave impinging upon a rough surface under impedance boundary condition (IBC), which means that the rough surface is highly-conducting. This work focuses on the two-dimensional rough surfaces, and uses the method of Moments as the numerical method. Finally, we compare the results with different permittivities and incident angles. It is helpful for the depth study of detecting the object above the real sea surface.
\end{abstract}

Key words - scattering of electromagnetic waves; highly-conducting; rough surfaces; Method of Moments

\section{INTRODUCTION}

Recently, research in the domain of electromagnetic scattering from randomly rough surfaces has been attracting more and more attention, due to its extensive applications in the fields of meteorology, navigation, oceanography, environment, military and many other important areas. This study is concerned about scattering from a sea surface, all the works are based on the numerical method -- the method of moments (MoM).

The method of moments is a kind of numerical method which is based on discrete the integral equations, and using integral equations have their own priority: First, due to the introduction of the Green function, the radiation condition of the electromagnetic field at infinity has been analytically included in the integral equation, so the relationship between unknowns can be accurately obtained to avoid numerical dispersion; Second, the number of unknowns generated by the integral equation are much less than that generated by the differential equations, so it is more applicable to calculate the electromagnetic scattering of a large target. In fact, this method is the key part of work.

According to the Maxwell equations and boundary conditions, the equation of the rough surfaces scattering is derived, this equation is discretized by the method of moments. That is to turn the solution of integral equations into matrix equations for solving. We can calculate the electromagnetic field or surface electromagnetic currents from the integral equations. Then, we can compute the far electromagnetic field from the Huygens principle. The key here is to solve the integral equations to calculate the currents.

Now this paper is devoted to the two-dimensional rough surfaces in the case of Highly-Conducting rough surfaces, we can obtain the currents from the integral equation, and get the scattered field from the IBC surface. Finally, we will compare the results with different permittivities and incident angles.

\section{SURFACE INTEGRAL EQUATION}

\section{A. Integral Equation}

A rough surface $S$ separates vacuum from a semi-infinite homogeneous medium. This medium, assumed to be non-magnetic, is characterized by its complex relative permittivity $\varepsilon_{r} . S$ is assumed to be twice continuously differentiable, and its unit normal vector $n$ is directed toward vacuum. The electromagnetic field is time harmonic, and an $\exp (-i w t)$ time dependence implies $g_{0}=-\exp \left(i k_{0} R\right) / 4 \pi R$ in vacuum, with wave number $k_{0}=\omega \sqrt{\varepsilon_{0} \mu_{0}}$, and $g=-\exp (i k R) / 4 \pi R$ below $S$, with wave number $k=k_{0} \sqrt{\varepsilon_{r}}$, where $R$ denotes the distance between the two points $r, r^{\prime}$. $j=n \times H \quad$ and $m=n \times E$ denote the electric and the magnetic surface currents respectively, unknowns of the Stratton-Chu equations[2].

From the Stratton-Chu formulas, applying the second Green's vector identity the electric field at a point in vacuum and taking the limit as this point goes onto the surface, and then from a series of Derivations, we can obtain the integral equation:

$$
\left[\left(I+\bar{M}_{0}-\bar{M}\right)-\frac{i}{\omega \mu_{0}} \overline{\left(P_{0}-P\right) \bar{Z}}\right] j=n \times H^{i}
$$

Since the magnetic current is weak, Eq.(1) is written as a modified magnetic field integral equation (MFIE), with an extra corrective term that represents finite conductivity[2]:

$$
\left(\frac{I}{2}+\bar{M}_{0}\right) j+\left\{\frac{I}{2}-\bar{M}-\frac{i}{\omega \mu_{0}} \overline{\left(P_{0}-P\right) \bar{Z}}\right\} j=n \times H^{i}
$$


$\bar{M}_{0}, \bar{M}$ and $\bar{P}_{0}, \bar{P}$ are the integral operators introduced by Martin and Ola in [3].

The impedance approximation leads to resolutions with approximately the same numerical cost as perfectly conducting surfaces: the number of unknowns and matrix size are unchanged, and only the matrix elements are more complicated and thus take longer time to compute.

\section{B. Integral Operators}

Here, considering the surface $S$ has the cartesian equation $z=f(x, y)$. $z_{x}=\frac{\partial f}{\partial x}(x, y), z_{y}=\frac{\partial f}{\partial y}(x, y)$ are the first derivatives of $z=f(x, y)$, and the second derivatives are $\quad z_{x x}=\frac{\partial^{2} f}{\partial x^{2}}(x, y)$, $z_{x y}=\frac{\partial^{2} f}{\partial x \partial y}(x, y), \quad z_{y x}=\frac{\partial^{2} f}{\partial y \partial x}(x, y)$, $z_{y y}=\frac{\partial^{2} f}{\partial y^{2}}(x, y)$.

\section{- Integral Operator $\bar{Z}$}

As a part of the impedance approximation, the operator $\bar{Z}$ such that $m=\bar{Z} j$ is local. The diagonal elements of $\bar{Z}$ are expressed as follows:

$$
\begin{aligned}
& \bar{Z}=\left(\begin{array}{ll}
\bar{Z}_{x x, i i} & \bar{Z}_{x y, i i} \\
\bar{Z}_{y x, i i} & \bar{Z}_{y y, i i}
\end{array}\right)=-i \eta_{0} k_{0} d\{(1- \\
& \left.\left.\frac{d}{2} \operatorname{tr}\left(\bar{S}_{i}\right) \bar{N}_{i}+d \bar{N}_{i} \bar{S}_{i}\right)\right\}
\end{aligned}
$$

$\overline{N_{i}}$ is the matrix of the vector product by normal. If the matrix of the extrinsic curvature tensor $\overline{\overline{S_{i}}}$ has a complex expression, the product $\overline{N_{i}} \overline{S_{i}}$ is simpler and involves only the second derivatives of $Z$. Its trace $\operatorname{tr}\left(\overline{S_{i}}\right)$ equals the sum of the surface's main curvatures.

with

$$
\begin{array}{r}
\overline{N_{i}}=\frac{1}{\varphi_{i}}\left(\begin{array}{cc}
-z_{x, i} z_{y, i} & -1-z_{y, i}^{2} \\
1+z_{x, i}^{2} & z_{x, i} z_{y, i}
\end{array}\right) \\
\overline{N_{i} S_{i}}=\frac{1}{\varphi_{i}}\left(\begin{array}{cc}
-z_{x y, i} & -z_{y y, i} \\
z_{x x, i} & z_{x y, i}
\end{array}\right)
\end{array}
$$

$\operatorname{tr}\left(\overline{S_{i}}\right)=\frac{1}{\varphi_{i}^{3}}\left[\left(1+z_{y, i}^{2}\right) z_{x x, i}-2 z_{x, i} z_{y, i} z_{x y, i}+\left(1+z_{x, i}^{2}\right) z_{y y, i}\right]$

Evaluated at the center of each subdomain $i=1, \ldots, N$.

- Integral Operator $\bar{M}_{0}$

Now, let us consider the matrix $\bar{M}_{0}$.

diagonal elements $(i \neq j)$ are null for submatrices $\bar{M}_{0 x x i i}$ and $\bar{M}_{0 y x i i}$ and are equal for submatrices $\bar{M}_{0 x x i i}$ and $\bar{M}_{0 y x i i}$ :

$$
\begin{gathered}
\bar{M}_{0 x x, i i}=\bar{M}_{0 x y, i i}=\frac{1}{8 \pi}\left(\iint_{D} \frac{x^{2}}{R^{3}} d x d y+\right. \\
\left.2 z_{x y, i} \iint_{D} \frac{x y}{R^{3}} d x d y+z_{y y, i} \iint_{D} \frac{y^{2}}{R^{3}} d x d y\right)
\end{gathered}
$$

For this expression, derivatives of order higher than 2 have been neglected, and only leading terms of the series expansion of the Green's function have been considered. Cauchy principal-value integrals can be analytically evaluated if the surface is approximated by its tangential plane at point $i$, i.e.,

$$
r=\left[x^{2}+y^{2}+\left(z_{x, i} x+z_{y, i} y\right)^{2}\right]^{2} \text {. In my }
$$

program, I calculate this integral equation using MAPLE, which is a system for doing mathematics on the computer.

\section{- Integral Operator $\bar{M}$}

$\bar{M}$ having diagonal submatrices, diagonal elements are evaluated by analytical integration of the kernel over the entire $x y$ plane. This gives

$$
\left(\begin{array}{ll}
\bar{M}_{x x, i j} & \bar{M}_{x y, i j} \\
\bar{M}_{y x, i j} & \bar{M}_{y y, i j}
\end{array}\right)=-\frac{i}{4 k} \frac{z_{x x, i}+z_{y y, i}}{\varphi_{i}}\left(\begin{array}{ll}
1 & 0 \\
0 & 1
\end{array}\right)
$$

- Integral Operator $\bar{\Delta}_{p}$

$\bar{\Delta}_{p}$ is equal to $\overline{P_{0}-P}$ which is handled globally to avoid hypersingularity. Nondiagonal elements of $\overline{P_{0}-P}$ are set equal to the product of the value at the subdomain center by the subdomain area $\Delta$ :

$$
\begin{aligned}
& \left(\begin{array}{ll}
\left({\overline{\left(P_{0}-P\right.}}_{x x, i j}\right. & {\overline{\left(P_{0}-P\right)_{x y, j}}}_{\left(\bar{P}_{0}-P\right)_{y x, j}} \\
\left(P_{0}-P\right)_{y, j, j}
\end{array}\right)=\iint_{D}\left\{\frac { N _ { 1 } ( R _ { i j } ) } { R _ { i j } } \left(\bar{A}_{10}+x \bar{A}_{x}+y \bar{A}_{y}\right.\right. \\
& \left.\left.+x^{2} \bar{A}_{1 x}+x y \bar{A}_{x y}+y^{2} \bar{A}_{(y)}\right)+N_{2}\left(R_{i j}\right) \bar{A}_{2}\right\} d x d y
\end{aligned}
$$

with the following notation: 


$$
\begin{aligned}
& \bar{A}_{10}=\left[\begin{array}{cc}
-\bar{T}_{10} \bar{T}_{30} & -\bar{T}_{20} \bar{T}_{30} \\
\bar{T}_{10} \bar{T}_{40} & \bar{T}_{20} \bar{T}_{40}
\end{array}\right] \\
& \bar{A}_{x}=\left[\begin{array}{cc}
\bar{T}_{10} \bar{T}_{3 x}+\bar{T}_{1 x} \bar{T}_{30} & \bar{T}_{20} \bar{T}_{3 x}+\bar{T}_{2 x} \bar{T}_{30} \\
-\left(\bar{T}_{10} \bar{T}_{4 x}+\bar{T}_{1 x} \bar{T}_{40}\right) & -\left(\bar{T}_{10} \bar{T}_{4 x}+\bar{T}_{1 x} \bar{T}_{40}\right)
\end{array}\right] \\
& \bar{A}_{y}=\left[\begin{array}{cc}
\bar{T}_{10} \bar{T}_{3 y}+\bar{T}_{1 y} \bar{T}_{30} & \bar{T}_{20} \bar{T}_{3 y}+\bar{T}_{2 y} \bar{T}_{30} \\
-\left(\bar{T}_{10} \bar{T}_{4 y}+\bar{T}_{1 y} \bar{T}_{40}\right) & -\left(\bar{T}_{10} \bar{T}_{4 y}+\bar{T}_{1 y} \bar{T}_{40}\right)
\end{array}\right] \\
& \bar{A}_{1 x}=\left[\begin{array}{ll}
-\bar{T}_{1 x} \bar{T}_{3 x} & -\bar{T}_{2 x} \bar{T}_{3 x} \\
\bar{T}_{1 x} \bar{T}_{4 x} & \bar{T}_{2 x} \bar{T}_{4 x}
\end{array}\right] \\
& \bar{A}_{1 y}=\left[\begin{array}{ll}
-\bar{T}_{1 y} \bar{T}_{3 y} & -\bar{T}_{2 y} \bar{T}_{3 y} \\
\bar{T}_{1 y} \bar{T}_{4 y} & \bar{T}_{2 y} \bar{T}_{4 y}
\end{array}\right] \\
& \bar{A}_{2}=\left[\begin{array}{cc}
-z_{y, i} z_{x, j} & 1-z_{y, i} z_{y, j} \\
1+z_{x, i} z_{x, j} & z_{x, i} z_{y, j}
\end{array}\right]
\end{aligned}
$$

the elements of the matrices are:

$$
\begin{aligned}
& \bar{T}_{10}=x_{i j}+z_{i j} z_{x, i} \bar{T}_{1 x}=1+z_{x, j}^{2}, \\
& \bar{T}_{1 y}=z_{x, j} z_{y, j}=\bar{T}_{2 x} \\
& \bar{T}_{20}=y_{i j}+z_{i j} z_{y, i}, \bar{T}_{2 x}=z_{x, j} z_{y, j}, \\
& \bar{T}_{2 y}=1+z_{y, j}^{2} \\
& \bar{T}_{30}=y_{i j}+z_{i j} z_{y, i} \bar{T}_{1 x}=1+z_{x, j}^{2}, \\
& \bar{T}_{1 y}=z_{x, j} z_{y, j}=\bar{T}_{2 x} \\
& \bar{T}_{40}=x_{i j}+z_{i j} z_{x, i}, \bar{T}_{4 x}=1+z_{x, j} z_{x, j}, \\
& \bar{T}_{4 y}=z_{x, i} z_{y, j}
\end{aligned}
$$

For the diagonal elements, the semi-analytical integrations give :

$$
\begin{aligned}
& \left(\begin{array}{ll}
{\overline{\left(P_{0}-P\right)_{x x, i j}}}_{\left(P_{0}-P\right)_{y x, i j}} & {\overline{\left(P_{0}-P\right)}}_{x y, i j} \\
\hline y, i j
\end{array}\right)= \\
& -2 \varphi_{i}^{2}\left(\int_{\frac{\Delta_{y}}{\Delta_{x}}}^{\frac{\Delta_{x}}{\Delta_{x}}}\left(\begin{array}{cc}
-v & 1 \\
-v^{2} & v
\end{array}\right) \frac{A_{1 x}(v)}{q_{x}^{5}(v)} d v+\int_{\frac{\Delta_{y}}{\Delta_{x}}}^{\frac{\Delta_{y}}{\Delta_{x}}}\left(\begin{array}{cc}
-v & v^{2} \\
-1 & v
\end{array}\right) \frac{A_{1 y}(v)}{q_{y}^{5}(v)} d v\right) \\
& -2\left(\int_{-\frac{\Delta_{y}}{\Delta_{x}}}^{\frac{\Delta_{y}}{\Delta_{x}}} \frac{A_{3 x}(v)}{q_{x}^{3}(v)} d v+\int_{-\frac{\Delta_{y}}{\Delta_{x}}}^{\frac{\Delta_{y}}{\Delta_{x}}} \frac{A_{y y}(v)}{q_{y}^{3}(v)} d v\right)\left(\begin{array}{cc}
-z_{x, i} Z_{y, i} & -\left(1+z_{y, i}^{2}\right) \\
1+z_{x, i}^{2} & z_{x, i} Z_{y, i}
\end{array}\right)
\end{aligned}
$$

with

$$
\begin{aligned}
& q_{x}(v)=\sqrt{1+v^{2}+\left(z_{x}+z_{y} v\right)^{2}} \\
& q_{x}(v)=\sqrt{v^{2}+1+\left(z_{x} v\right)^{2}+z_{y}}
\end{aligned}
$$

$$
\begin{aligned}
& A_{1}(v)=\frac{1}{4 \pi}\left\{2 i\left(k_{0}-k\right) q(v)+\left(i k_{0} q(v)-\right.\right. \\
& \left.\left.\frac{6}{\Delta}\right) \exp \left(i k_{0} q(v) \frac{2}{\Delta}\right)-\left(i k q(v)-\frac{6}{\Delta}\right) \exp \left(i k q(v) \frac{\Delta}{2}\right)\right\} \\
& A_{3}(v)=\frac{1}{4 \pi}\left\{2 i\left(k_{0}-k\right) q(v)-\frac{4}{\Delta} \exp \left(i k_{0} q(v) \frac{\Delta}{2}\right)\right. \\
& \left.+\frac{4}{\Delta} \exp \left(i k q(v) \frac{\Delta}{2}\right)\right\}
\end{aligned}
$$

Where we put $q_{x}$ and $\Delta_{x}$ into $A_{1}$ instead of $q$ and $\Delta$, then we can get the equation of $A_{1 x}$, and use the same way to obtain the equations of $A_{1 x}, A_{2 x}$ and $A_{3 x}$.

\section{RESUlTS AND DisCUSSION}

After calculating all the operators, we can get the impedance approximation matrix. Then we can obtain the currents from the integral equation, and get the scattered field from the IBC surface. Here, we will compare the results which we obtain from MATLAB with different permittivities and incident angles.

\section{A. Case of a Smooth Plate}

First, let us consider the case of a highly-conducting plate as shown in Fig.1. An electromagnetic plane wave with the incident angle $\theta_{i}$. impinging upon the center of a plate, as we can see from Fig.1, $\theta_{s}$ is the scattering angle. Here we define $\lambda=1 \mathrm{~m}$, the dimension of the plate are $l_{x}=6 \lambda, l_{y}=6 \lambda$, and it is discretized with a sampling steps of $\Delta_{x}=\Delta_{y}=\frac{\lambda}{8}$. The polarisation vectors are also shown in the figure. In this step, we choose four different permittivities $10+10 \mathrm{j}, 20+20 \mathrm{j}, 30$ $+30 \mathrm{j}, 40+40 \mathrm{j}$.

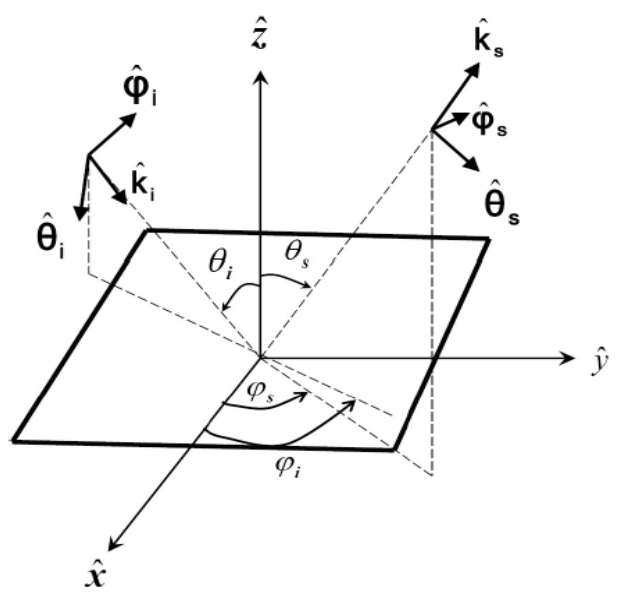

Fig.1. Wave imping upon a highly-conducting plate 
When the incident angle $\theta_{i}=0^{\circ}$, $\varphi_{i}=0^{\circ}$ and $\varphi_{s}=0^{\circ}$, seeing from Fig.2, The horizontal axis represents the range of the observation angles in degree, and the vertical axis indicates the RCS in $d B m^{2}$. The RCS reaches maximum at the $\theta_{s}=0^{\circ}$ for the four different permittivities, it is the specular direction $\left(\theta_{i}=\theta_{s}\right)$ and the maximum value decreases while the permittivity increases. This result is from MATLAB.

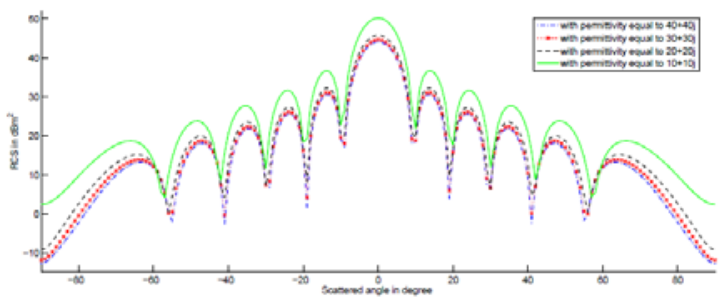

Fig.2. Radar Cross Section in $\mathrm{dBm}^{2}$ of a IBC rough surface

When the incident angle is $30^{\circ}$, as shown in Fig.3, the RCS reaches maximum at the $\theta_{s}=-30^{\circ}$ for the four different permittivities, it is still the specular direction and the maximum values decrease while the permittivity increases.

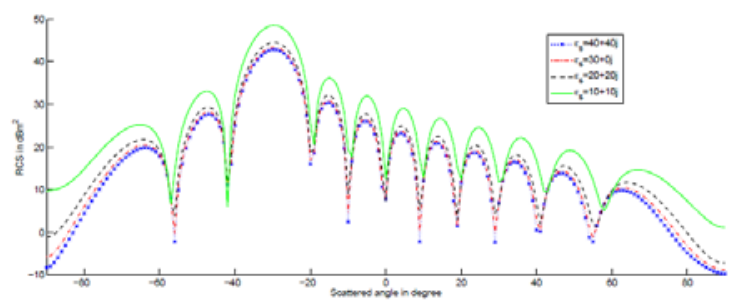

Fig.3. Radar Cross Section in $d B m^{2}$ of a IBC rough surface

We continue to put the incident angle $\theta_{i}=60^{\circ}$, as shown in Fig.4, the RCS reaches maximum at the $\theta_{s}=-60^{\circ}$ for the four different permittivities, and the maximum values decrease while the permittivity increases.

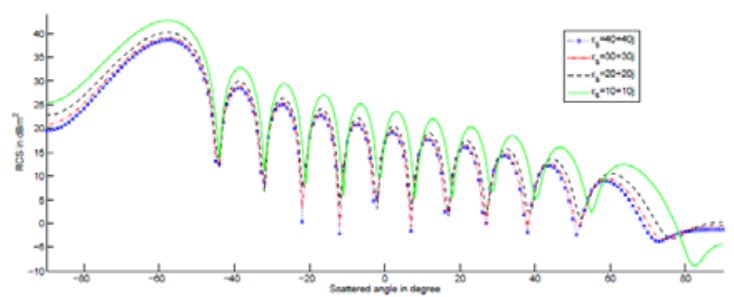

Fig.4. Radar Cross Section in $d \mathrm{Bm}^{2}$ of a IBC rough surface

We can conclude that RCS reaches maximum at the specular direction for a plate.

\section{B. Case of a Rough Surface}

We consider the case of highly-conducting rough surface as shown on the Fig.5, dimensions and sampling steps are the same with the case of a plate. For the rough surface, the height root mean square $\sigma_{h}=0.2 \lambda$, correlation length $l_{c x}=1.2 \lambda, l_{c y}=1.2 \lambda$. Correlation function of 2-D rough surface satisfies:

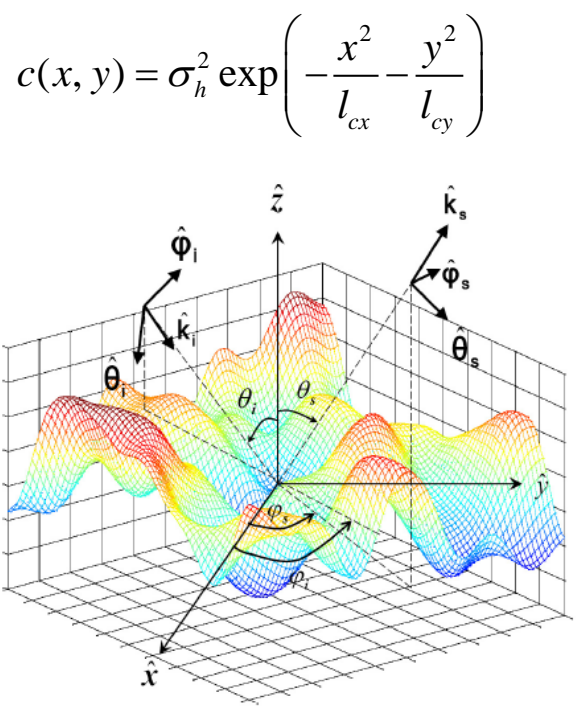

Fig.5. Wave imping upon a highly-conducting rough surface

When the incident angle $\theta_{i}=0^{\circ}$, Fig. 6 shows that RCS reaches the maximum at $\theta_{s}=10^{\circ}$ for the four permittivities. The maximum value increases while the permittivity increases. Because if the permittivity is high, the field strength decline in the dielectric, so the value of RCS is higher for a high permittivity rough surface.

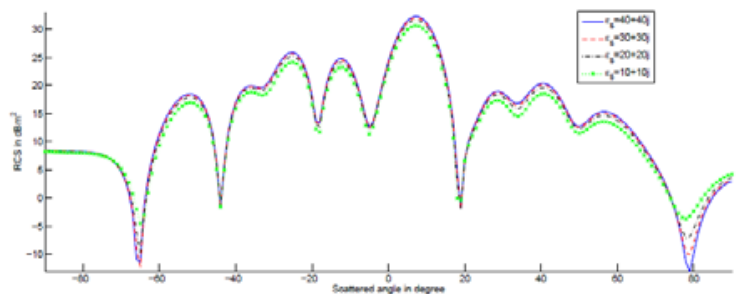

Fig.6. Radar Cross Section in $\mathrm{dBm}^{2}$ of a IBC rough surface

When the incident angle $\theta_{i}=30^{\circ}$, Fig.7 shows that RCS reaches the maximum at $\theta_{s}=-23^{\circ}$ for the four cases. The maximum values still increases while the permittivity increases. 


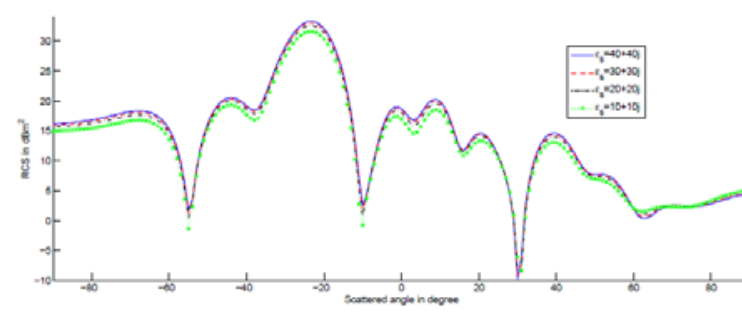

Fig.7. Radar Cross Section in $d \mathrm{Bm}^{2}$ of a IBC rough surface

When the incident angle $\theta_{i}=60^{\circ}$, Fig.8 shows that RCS reaches the maximum at $\theta_{s}=-58^{\circ}$ for the four permittivities, the maximum value still increases while the permittivity increases.

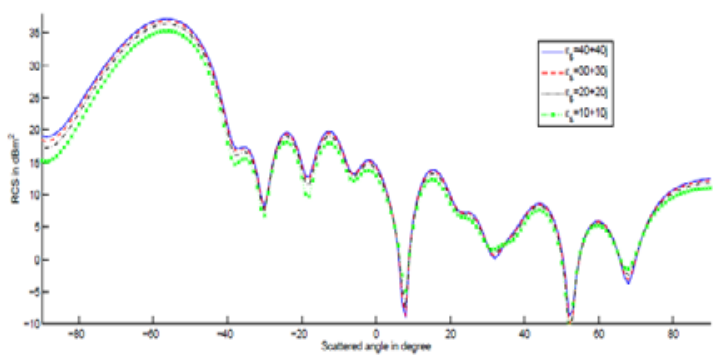

Fig.8. Radar Cross Section in $d B m^{2}$ of a IBC rough surface

\section{CONCLUSION}

In this report, the scattered field by impedance boundary condition (IBC) rough surface is studied. From the results, we can see that the scattered field by a rough surface is more complicated than the smooth plate, the power of the scattered field by a smooth plate is higher than the rough surface, this means that there has power lost by a rough surface. Mainly because the power distribution of the rough surface is more dispersed, so the maximum value of the power is reduced, but the total field is unchanged. Comparing the figures, we can also see that the maximum value increases while the permittivity increases for the rough surface, but for the plate, the situation is exactly the opposite, and we can find that the results are not very satisfied for a plate. The problem maybe come from the integral equation which uses to calculate the operators, or others, so more work should be done in the future.

\section{ACKNOWLEDGMENT}

First, I want to thank my tutor Professor Christophe Bourlier, he gives me directive suggestions, so that I can complete my paper successfully. And then I would also like to thank my colleague Professor Shao Yufeng and Jiancun Zuo , they give me a lot of help when I write this paper. Finally, I want to thank all the people who help me when I write the paper.

And then, this work is partially supported by the National Natural Science Foundation of
China (No. 61107064), Innovation Program of Shanghai Municipal Education Commission (No. 15ZZ101), Leading Academic Discipline Project of Communication and Information System (No. XXKZD1302), Youth Teacher Training Program of Shanghai University(No. ZZegd14003) and School Foundation (No.EGD14XQD01) of Shanghai Second Polytechnic University.

\section{REFERENCES}

[1] Y.Huang, "The numerical methods of electromagnetic scattering rough surface "[D]. Huazhong University of Science and Technology,2008.

[2] G.Soriano and M.Saillard, "Scattering of electromagnetic waves from two-dimensional rough surfaces with an impedance approximation”, J.Opt.Soc.Am.A/Vol.18, No.1/January 2001.

[3] P.A.Martin and P.Ola, "Boundary integral equations for the scattering of electromagnetic waves by a homogeneous dielectric obstacle”, Proc.Roy.Soc, Edinburgh, 123A, 185-208(1993).

[4] P.Tran and A.A.Maradudin, "The scattering of electromagnetic waves from a randomly rough 2D metallic surface”, Optics Comm, 110:269-273, August 1994.

[5] A.M.Matvin, V.Celli, "Relation between the surface impedance and the

extinction theorem on a rough surface", Phys.Rev.B50, 14546-14553(1994).

[6] K.Pak, L.Tsang, C.H.Chan, and J.T.Johnson,"Back-scattering enhancement of electromagnetic waves from two-dimensional perfectly conducting random rough surfaces based on Monte Carlo simulations”, J.Opt.Soc.Am.A12, 2491-2499(1997).

[7] C.Macaskill and B.J.Kachoyan, "Iterative approach for the numerical simulation of scattering from one and two dimensional rough surfaces”, Applied Optics, 32:2839-2847, 1993.

[8] P.Tran, V.Celli, and A.A.Maradudin, "Electromagnetic scattering from a two-dimensional, randomly rough perfectly conducting surface: iterative methods”,J.Opt.Soc.Am.A11,1886-1889(1994). 